Casos Clínicos

Arch. Esp. Urol., 61, 4 (524-527), 2008

\section{METÁSTASIS RETROORBITARIA EN EL DIAGNÓSTICO INICIAL DEL CÁNCER DE PRÓSTATA}

Héctor Pastor Navarro, María José Donate Moreno, Pedro Carrión López, Jesús Martínez Ruiz, Juan Gabriel Lorenzo Romero, José Miguel Jiménez Bachs, Lorenzo Polo Ruiz, Antonio S. Salinas Sanchez y Julio A. Virseda Rodríguez.

Servicio de Urología. Hospital General Universitario de Albacete. Albacete. España.

Resumen.- OBJETIVO: Describir un caso de cáncer prostático avanzado que debutó como primera manifestación clínica con una diplopia binocular por metástasis retroorbitaria.

MÉTODOS: Presentamos un paciente de 54 años que es diagnosticado de cáncer prostático diseminado tratado mediante bloqueo androgénico completo.

RESULTADOS: Tras la instauración del tratamiento hormonal el paciente mejoró inicialmente si bien la supervivencia fue limitada.

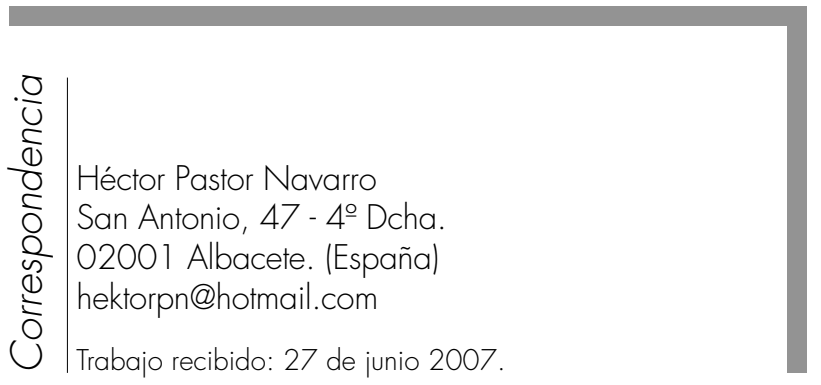

CONCLUSIONES: La metástasis orbitaria es una localización infrecuente dentro de la diseminación tumoral del cáncer de próstata que implica una supervivencia limitada que oscila entre 7,5-30 meses. En nuestro caso fue de 10 meses desde el diagnóstico.

Palabras clave: Adenocarcinoma prostático. Metástasis retroorbitaria.

Summary.- OBJECTIVE: To describe one case of advanced prostate cancer first presenting with binocular diplopia due to retroorbital metastasis.

METHODS: We present the case of a 54-year-old patient with the diagnosis of disseminated prostate cancer treated by complete androgen blockade.

RESULTS: After the start of hormonal treatment, the patient initially improved although survival was limited.

CONCLUSIONS: Orbital metastasis is an infrequent site for prostate cancer dissemination which implies a limited survival oscillating between 7,5-30 months. In our case survival was 10 months from diagnosis.

Keywords: Prostatic adenocarcinoma. Retroorbital metastasis.

\section{INTRODUCCIÓN}

El cáncer prostático es la neoplasia urogenital más frecuente y supone la tercera causa de muerte por cáncer en el varón tras el pulmonar y colorrectal. Entre un 20$30 \%$ de pacientes que son diagnosticados de adenocarcinoma prostático presentan enfermedad localmente avanzada o metastásica (1) debido fundamentalmente a la escasa sensibilidad de los métodos utilizados habitualmente para el screening.

Las metástasis habituales en el adenocarcinoma prostático afectan a adenopatias pélvicas (territorio de vasos iliacos internos y externos y fosa obturatriz) y al esqueleto axial, siendo poco frecuentes las intracraneales.

\section{CASO CLÍNICO}

Paciente varón de 54 años de edad sin antecedentes personales de interés.

Acude al Servicio de Urgencias de nuestro hospital en septiembre de 2005 por cefalea frontoparietal derecha 
y diplopia de mirada lateral de 2 semanas de evolución así como edema palpebral derecho desde hace una semana.

Fue valorado inicialmente por el Servicio de Oftalmología objetivando exoftalmos moderado en ojo derecho, inflamación, hiperemia y tumefacción palpebral superior e inferior derecha moderadas, limitación en todas las posiciones de de la mirada y diplopia. No presentaba alteración de la agudeza visual. La presión intraocular (PIO) del ojo derecho era de $20 \mathrm{mmHg}$., la PIO del ojo izquierdo fue de $10 \mathrm{mmHg}$. La exploración del fondo de ojo era normal.

Se solicitó TAC orbitario (Figura 1) objetivándose aumento de tamaño del hueso esfenoides con patrón permeativo e hiperostosis de cara orbitaria con masa de partes blandas que abombaba el músculo recto lateral. Asimismo se producía ocupación de las celdillas etmoidales.

El paciente ingresó en el Servicio de Neurocirugía solicitándose RMN craneal que era informado como posible meningioma hiperostósico en placa periorbitaria derecha del ala mayor de esfenoides sin poder descartar lesiones fibroóseas esfenoidales.

Se programó para realizar biopsia quirúrgica de dichas lesiones. El resultado anatomopatológico de la biopsia fue de tejido conectivo, músculo esquelético estriado y aisladas trabéculas óseas con nidos y cordones de células epiteliales con citoplasma amplio que formaban focalmente luces glandulares, en el hueso se apreciaba metástasis de adenocarcinoma con positividad inmunohistoquímica para el Antígeno Prostático Específico (PSA).

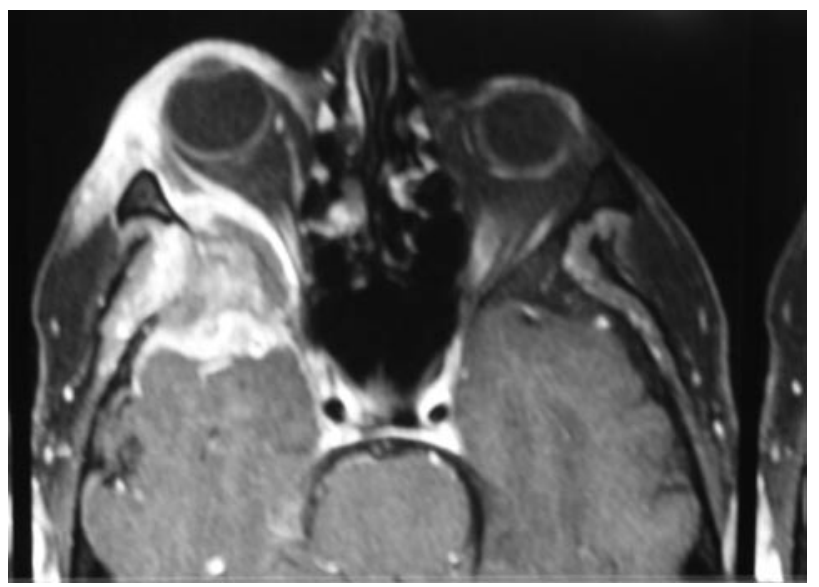

FIGURA 1. TAC craneal. Aumento de tamaño del hueso esfenoides con patrón permeativo e hiperostosis de cara orbitaria con masa de partes blandas que abomba músculo recto lateral. Ocupación de celdillas etmoidales.
Ante estos hallazgos se consultó con el Servicio de Urología. Realizando una anamnesis dirigida se descubrieron alteraciones en la micción en los últimos meses (polaquiuria y nocturia) y hemospermia.

\section{Exploración física}

Tacto rectal: próstata volumen II/IV, de consistencia pétrea en ambos lóbulos, fija, sospechosa de cáncer de próstata.

\section{Pruebas complementarias}

PSA: $389 \mathrm{ng} / \mathrm{ml}$

Biopsia de próstata transrrectal: adenocarcinoma prostático Gleason $4+4=8$ que afecta extensamente a ambos lóbulos.

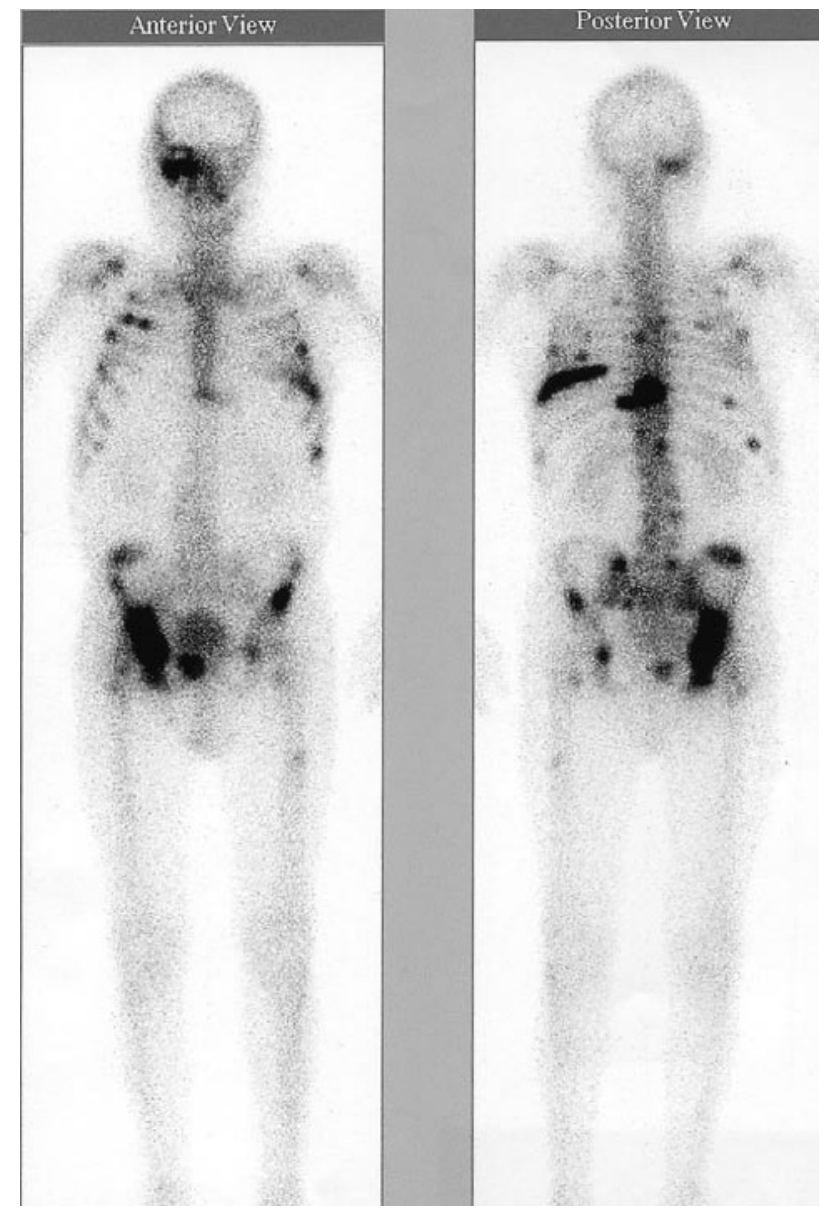

FIGURA 2. Gammagrafía que revela deseminación ósea metastática. Obsérvese en la visión anterior la metástasis orbitaria derecha. 
Rastreo óseo con Tc99m (Figura 2): las imágenes mostraban múltiples áreas de hipercaptación que afectan a región cigomática derecha y orbital interna derecha, ambas parrillas costales, columna dorsolumbar, pala ilíaca, región acetabular e isquiopubiana derechas, espina iliaca y región acetabular izquierdas y tercio medio de diáfisis femoral izquierda.

\section{Diagnóstico}

Adenocarcinoma de próstata Gleason $4+4=8$ con metástasis óseas múltiples.

\section{Tratamiento}

Se instauró tratamiento con bloqueo hormonal completo (Bicalutamida $50 \mathrm{mg} / 24 \mathrm{~h}$ y Goserelina $10,8 \mathrm{mg} / 12$ semanas) y bifosfonatos (Ácido Zoledrónico $4 \mathrm{mg}$ iv /4 semanas).

\section{Evolución}

Tras la instauración del bloqueo androgénico se produjo una mejoría progresiva de la clínica ocular. Durante los primeros 7 meses de seguimiento el paciente se mantuvo asintomático.

A los 8 meses el paciente ingresó en el Servicio de Urología con clínica de hematuria, que al no mejorar con lavado vesical continuo, requirió la realización de RTU vesical, apreciándose gran proceso neoformativo prostático que invadía el suelo vesical. Anatomía patológica de los fragmentos de resección: focos de adenocarcinoma prostático con cambios involutivos secundarios a tratamiento hormonal.

A los 9 meses del diagnóstico inicial el paciente ingresa de nuevo en nuestro servicio por síndrome miccional obstructivo, disminución de la diuresis, deterioro de la función renal y disnea. Se realizó TAC toracoabdominopélvico apreciándose derrame pleural derecho con atelectasia compresiva, múltiples adenopatías mediastínicas, metástasis pulmonares múltiples, dilatación ureteropielocalicial del riñón izquierdo y las ya conocidas múltiples lesiones óseas metastásicas de tipo blástico.

Se realizó pleurocentesis derecha (la citología del líquido pleural fue positiva para células tumorales malignas). Se desestimó la colocación de nefrostomía percutánea.

El paciente pasa a ser controlado por la Unidad de Cuidados Paliativos. Falleció 10 meses después del diagnóstico inicial.

\section{DISCUSIÓN}

El adenocarcinoma de próstata es la neoplasia urogenital más frecuente, constituye la tercera causa de muerte por cáncer en el varón. Alrededor de un 20-30\% de los pacientes presentan en el momento del diagnóstico tumores localmente avanzados o metastáticos (1-3).

Las metástasis del adenocarcinoma prostático se localizan con mayor frecuencia en los ganglios linfáticos pélvicos y en el esqueleto óseo, generalmente en pelvis, columna, fémur y costillas en orden decreciente de frecuencia. Suelen ser lesiones osteoblásticas en el 66$90 \%$ de los casos (4).

Las metástasis orbitarias son una entidad inusual en adultos representando solamente el $2-9 \%$ de los tumores orbitarios (5). El origen más frecuente de metástasis orbitaria es la mama, el pulmón, la próstata, el melanoma y el riñón (6). Una metástasis orbitaria de adenocarcinoma prostático suele ser muy raro encontrarla como manifestación inicial de la enfermedad (7).

La vía de diseminación de las metástasis en en adenocarcinoma prostático suele ser linfática y hematógena. La diseminación hematógena se produce a través del plexo venoso prevertebral de Batson (8) (lo que justifica que la afectación ósea sea fundamentalmente axial) por dónde llegaría a las leptomeninges, constituyendo la localización metastásica intracraneal más frecuente. Las metástasis orbitarias se producen por vía arterial, por medio de émbolos tumorales que atraviesan el filtro pulmonar (6).

Los síntomas de presentación de una metástasis orbitaria suelen ser disminución de la agudeza visual, proptosis, dolor ocular, diplopia, ptosis, cefalea, etc. $(9,10)$.

Debemos establecer diagnóstico diferencial con otros procesos expansivos intracraneales. Las pruebas de imagen, TAC y RMN son de gran importancia, con las que podremos visualizar la lesión y determinar su extensión. Sin embargo, no existe ningún dato radiológico específico que nos alerte de que se trata de un tumor metastático, ya que las lesiones osteoblásticas también se pueden presentar en otros procesos como por ejemplo los meningiomas $(2,3,5,10)$. Por lo tanto, la técnica más fiable para determinar el origen de la lesión será la biopsia. El estudio histopatológico mostrará cordones de células epiteliales atípicas que forman estructuras glandulares y que mostrarán positividad para el Antígeno Prostático Específico (PSA) (1-3, 5, 9).

Las modalidades de tratamiento incluyen quimioterapia, terapia hormonal, radioterapia y terapias combinadas. La decisión se tomará dependiendo del estadío evolutivo de la enfermedad y la situación general del paciente. La radioterapia externa, aislada o en combinación con la cirugía, ha sido eficaz en los pacientes donde la metástasis tiene poco tiempo de evolución y la enfermedad no está diseminada, que al igual que el bloqueo androgénico ha mejorado la calidad de vida del enfermo.

En nuestro caso, el tratamiento hormonal supresivo produjo una reducción completa de la masa retroorbitaria, 
no siendo necesario el tratamiento con radioterapia externa.

El pronóstico de los pacientes con enfermedad metastásica en la región orbitaria es desfavorable. Los estudios publicados en cuanto a la supervivencia son variables, estimándose entre 7,5-30 meses. En nuestro caso fue de 10 meses.

\section{CONCLUSIÓN}

Consideramos que el cáncer de próstata debería ser tenido en cuenta a la hora del diagnóstico diferencial de masas retrorbitarias de posible origen metastásico en pacientes con clínica neuro-oftalmológica, aún cuando el paciente no relate clínica urológica específica.

\section{BIBLIOGRAFIA Y LECTURAS RECOMENDADAS ( ${ }^{*}$ lectura de interés $y^{* *}$ lectura fundamental)}

1. PIERAS, E.; ROSALES, A.; LÓPEZ, H. y cols.: "Metástasis intracraneales en cáncer de próstata". Actas Urol. Esp., 24: 626, 2000.

2. FRANCO, E.; GIL-NÉCIJA, E.; CANO, G. y cols.: "Metástasis esfenoidal simulando meningioma como manifestación inicial de adenocarcinoma de próstata". Rev. Neurol., 29: 929, 1999.

3. BONILLO GARCÍA, M.A.; QUEIPO ZARAGOZÁ, J.A.; PALMERO MARTÍ, J.L. y cols.: "Metástasis retroorbitaria como manifestación inicial del cáncer de próstata". Actas Urol. Esp., 27: 562, 2003.

**4. SUTTON, M.A.; WATKINS, H.L.; GREEN, L.K. y cols.: "Intracranial metastases as the first manifestation of prostate cancer". Urology, 48: 789, 1996.

5. PRAT-BARTOMEU, J.: "Metástasis orbitarias en el adulto". Rev. Neurol., 31: 1261, 2000.

**6. BALTOGIANNIS, D.; KALOGEROPOULOS, C.; IOACHIM, E. y cols.: "Orbital metastasis from prostatic carcinoma”. Urol. Int., 70: 219, 2003.

7. GONZÁLEZ, C.; KUZEL, T.; CARTER, M.: "Metastatic adenocarcinoma of the prostate to the orbit as a presenting symptom". J. Urol., 157: 625, 1997.

8. BATSON, O.V.: "The function of the vertebral veins and their role in the spread of metastases". Annals of Surgery, 112: 138, 1940

9. AUBERT, J.; IRANI, J.: "Saint-Blancatp. Orbital metastasis of prostatic cancer. Clinical and therapeutic aspects. A propos of a case". Chirurgie, 121: 672, 1997.

10. CARRIERE, V.M.; KARCIOGLU, Z.A.; APPLE, D.J. y cols.: "A case of prostate carcinoma with bilateral orbital metastases and the review of the literature". Ophthalmology, 89: 402, 1982.

Casos Clínicos

Arch. Esp. Urol., 61, 4 (527-531), 2008

\section{LINFOMA TESTICULAR PRIMARIO. PRESENTACIÓN DE DOS CASOS Y REVISIÓN DE LA LITERATURA}

\author{
Paloma Delgado Bavai, Jorge Abad Roger, Araceli \\ Bono Ariño, Miguel Esclarin Duny, Miguel Marigil \\ Gomez y José Ignacio Sanz Velez.
}

Servicios de Urología y Anatomía Patológica'. Hospital San Jorge. Huesca. España.

Resumen.- OBJETIVO: Comunicación de dos casos clínicos con diagnóstico de linfoma testicular primario y revisión de la literatura existente sobre esta patología.

MÉTODOS/RESULTADOS: Descripción de dos casos de linfoma testicular primario en dos varones de 69 y 61 años. En ambos casos su motivo de consulta fue un aumento de volumen testicular derecho. Tras practicar una orquiectomía inguinal se diagnóstico, en el primer caso, un linfoma difuso tipo $T$ de células grandes y medianas, estadio I (E)-A, que tras seguir tratamiento con quimioterapia (R-CHOP) se mantiene en remisión a los tres años. En el segundo caso, el diagnóstico fue de linfoma difuso de células grandes

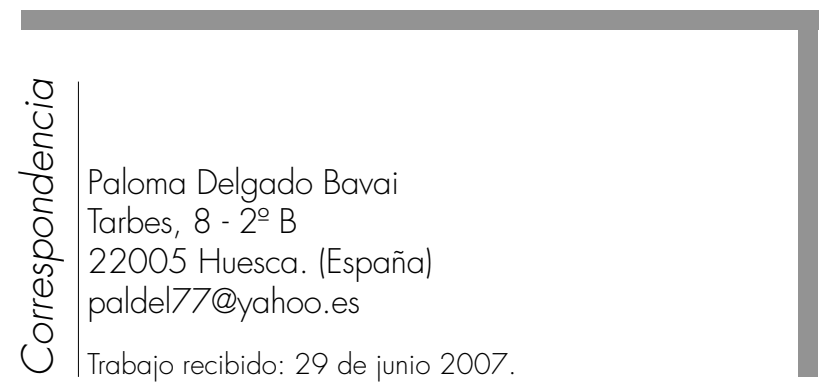

